\title{
The Role of Regular Physical Therapy on Spasticity in Children With Cerebral Palsy
}

\author{
Heewon Lee, MD ${ }^{1}$, Eun Kyung Kim, MA, PT ${ }^{1}$, Dong Baek Son, MS, PT ${ }^{1}$, Youngdeok Hwang, PhD², \\ Joon-Sung Kim, MD, PhD ${ }^{1}$, Seong Hoon Lim, MD, $\mathrm{PhD}^{1}$, Bomi Sul, $\mathrm{MD}^{1}$, Bo Young Hong, MD, $\mathrm{PhD}^{1}$
}

${ }^{1}$ Department of Rehabilitation Medicine, St. Vincent's Hospital, College of Medicine, The Catholic University of Korea, Suwon; ${ }^{2}$ Department of Statistics, Sungkyunkwan University, Seoul, Korea

Objective To investigate the effect of physical therapy (PT) intervention on spasticity in patients with cerebral palsy (CP), and to assess the degree of deterioration of spasticity when regular PT is interrupted in those patients. Methods We recruited 35 children with spastic CP who visited our hospital for PT, and whose Modified Tardieu Scale (MTS) scores were serially recorded including before and after a 10-day public holiday time frame period. The outcome measures were the angle of range of motion (ROM) of dorsiflexion of the ankle joint (R1 and R2) in the knee flexion and extension positions as assessed using the MTS.

Results The range of dorsiflexion of the ankle joint (R1 and R2) after the holiday period was significantly decreased as compared with that measured ROM noted before the holiday period, regardless of the knee position, age, or gross motor function. The dynamic component of the MTS (R2-R1) showed a slight decrease in the knee flexion position.

Conclusion Interruption of regular PT aggravated spasticity and decreased ankle joint ROM in children with spastic CP. Our findings suggest that regular PT in the care continuum for children with CP is crucial for the maintenance of ROM in the spastic ankle joints.

Keywords Cerebral palsy, Muscle spasticity, Physical therapy, Ankle joint, Range of motion

\section{INTRODUCTION}

Cerebral palsy (CP) is a group of developmental disorders of movement and posture that limit activity and are attributable to non-progressive disturbances that occurred in the developing fetal or infant brain. In these cases, the motor disorders of CP are often accompanied by impairments in sensation, cognition, communication, perception, behaviour, and a seizure disorder [1]. In this context, $\mathrm{CP}$ is classified into four subtypes: spastic

Received August 7, 2018; Accepted October 30, 2018

Corresponding author: Bo Young Hong

Department of Rehabilitation Medicine, St. Vincent's Hospital, College of Medicine, The Catholic University of Korea, 93 Jungbu-daero, Paldal-gu, Suwon 16247, Korea. Tel: +82-31-249-7650, Fax: +82-31-251-4481, E-mail: byhong@catholic.ac.kr

ORCID: Heewon Lee (http://orcid.org/0000-0001-8367-7575); Eun Kyung Kim (http://orcid.org/0000-0002-9429-2133); Dong Baek Son (http://orcid. org/0000-0001-9714-0517); Youngdeok Hwang (http://orcid.org/0000-0001-7563-5156); Joon-Sung Kim (http://orcid.org/0000-0001-7457-593X); Seong Hoon Lim (http://orcid.org/0000-0002-5475-4153); Bomi Sul (http://orcid.org/0000-0001-7166-8997); Bo Young Hong (http://orcid.org/00000001-9290-6173).

(c) This is an open-access article distributed under the terms of the Creative Commons Attribution Non-Commercial License (http://creativecommons.org/ licenses/by-nc/4.0) which permits unrestricted noncommercial use, distribution, and reproduction in any medium, provided the original work is properly cited. Copyright ( 2019 by Korean Academy of Rehabilitation Medicine 
(unilateral and bilateral), dyskinetic, ataxic, and nonclassifiable based on muscle tone and its nature of the condition, according to Surveillance of Cerebral Palsy: a European collaboration [2]. The spastic subtype, which is characterized by persistent increased muscle tone in one or more limbs, is the most common subtype and usually is known as accounting for $70 \%-90 \%$ of cases in children $[3,4]$.

Spasticity is generally defined as "a velocity-dependent increase in tonic stretch reflexes with exaggerated tendon jerks, resulting from hyperexcitability of the stretch reflex, as one component of the upper motor neuron syndrome" [5]. Spasticity presents various clinical signs of motor impairments including loss of voluntary muscle activation, abnormal involuntary muscle activation, and pain which associated with hip dislocation and contracture in the presence of gross motor function decline [3]. For this reason, spasticity is thought to cause changes in muscle morphology and structure, muscle deformity, and impaired muscle growth [6]. Furthermore, various foot deformities and secondary complications, such as hip displacement, may arise due to spasticity in children with this condition [7]. A previous study using the Ashworth scale to measure muscle tone in children with CP found that muscle tone tended to increase up to 4 years of age and then decrease each year up to 12 years of age [8]. Increased spasticity inhibits the stretching of muscles, which may cause musculoskeletal deformities leading to growth restriction and gait instability [9]. Thus, it is important to consider the early use of therapeutic interventions that focus on the control of muscle tone and prevention of muscle contracture are important for children with spastic CP.

Several approaches, including temporary medical interventions such as botulinum toxin injections and muscle relaxants; neurosurgical interventions such as selective dorsal rhizotomy; and permanent orthopedic interventions have been used to control spasticity and prevent secondary complications and pain related to musculoskeletal deformities in children with this condition [10-12]. However, exercise intervention has been the primary method of rehabilitation for hypertonia in patients with $\mathrm{CP}$ for several decades. To this end, patients with CP commonly undergo regular physical therapy (PT) stretching programs, which include passive and active stretching and prolonged positioning [13].
Evidence supporting the effectiveness of PT has accumulated over the past several decades; however, it is noted that the clinical outcomes vary considerably among patients, and the appropriate frequency and duration of stretching remains inconclusive. The purpose of our study was to provide evidence that regular PT was effective for patients with spastic CP. Because a prospective randomized study that stopped or interrupted PT was not possible for ethical reasons, we retrospectively reviewed progress notes and charts around the long public holiday period to investigate the role of $\mathrm{PT}$ in patients with $\mathrm{CP}$. We used the Modified Tardieu Scale (MTS) to assess changes in range of motion (ROM) before versus after the break in regular PT during the holiday period.

\section{MATERIALS AND METHODS}

In this study, the medical records of children with spastic CP who regularly participated in a PT program at our university hospital were retrospectively reviewed. Additionally, the PT programs were individualized according to the children's characteristics and functional level. In this sense, the neurodevelopmental therapy encompassing motor learning and goal-directed training concept was applied. For children who are not able to maintain standing posture independently, standing at the standing frame was usually attempted and performed, and stretching exercise was also incorporated in children with spasticity. The study was reviewed and approved by the Institutional Review Board of St. Vincent's Hospital (No. VC17RESI0204). The written informed consent was waived by the Institutional Review Board. The PT was discontinued during nearly the 10-day public holiday period between September and October 2017 for most children with CP in South Korea. We enrolled 35 children with spastic CP (19 boys, 16 girls) having serial MTS measurements, including before and after the 10-day holiday, performed by the same physical therapist at the beginning of each PT program. The CP subtype was classified according to the Surveillance of Cerebral Palsy in Europe guidelines [2] and the Gross Motor Function Classification System (GMFCS), a reliable and valid five-level classification system. The GMFCS levels are distinguished based on functional limitations and the need for assistive devices [14]. We reclassified the GMFCS levels into two groups for analysis: level I-III comprised children who 
were able to walk with or without aids, and level IV-V included children who were dependent on wheeled mobility [15].

We measured passive ROM and muscle spasticity in the ankle plantarflexor muscle for the reason that it is commonly involved in spasticity in the pediatric patient population. We used the MTS, a reliable tool for the measurement of spasticity, to assess the passive range of dorsiflexion of the ankle joint, where R2 was defined as the full ROM during a slow-velocity stretch, and R1 was defined as the threshold angle of catch-and-release or clonus at fast speed of stretch [16]. In fact, the ROM, R1, and R2 were measured in standardized knee flexion and knee extension positions. The ROM of the ankle joints was measured using a goniometer in $5^{\circ}$ increments. The MTS $\mathrm{R} 1$ and R2 values are commonly recorded in PT progress notes. We defined the neutral ankle position as $0^{\circ}$, and the dorsiflexion and the plantar flexion angles were converted to positive and negative values, respectively.

\section{Statistics}

A linear mixed model was used to assess the degree of change in MTS before versus after the public holiday break. The age, sex, GMFCS level, and collapsed GMFCS level (I-III vs. IV-V) were included as variables in the statistical analysis. Raw age data were analyzed as follows: $\leq 4$ years vs. $>4$ years and $\leq 5$ years vs. $>5$ years; this was

Table 1. Patients' characteristics $(n=35)$

\begin{tabular}{|cc}
\hline \multicolumn{1}{c}{ Characteristic } & Value \\
\hline Age (yr) & $5.6 \pm 2.2(1-11)$ \\
Gender & $19(54.3)$ \\
Male & $16(45.7)$ \\
\hline Female & \\
Cerebral palsy distribution & $13(37.1)$ \\
\hline Unilateral & $22(62.9)$ \\
\hline Bilateral & \\
\hline GMFCS level & $11(31.4)$ \\
\hline I & $4(11.4)$ \\
\hline II & $1(2.9)$ \\
\hline III & $9(25.7)$ \\
\hline IV & $10(28.6)$ \\
\hline V
\end{tabular}

Values are presented as mean \pm standard deviation or number (\%).

GMFCS, Gross Motor Function Classification System. based on a previous study in which muscle tone had a tendency to decrease in children with spastic CP older than 4 years [8]. All statistical tests were performed using $\mathrm{R}$ for Windows software (R Foundation for Statistical Computing, Vienna, Austria). p-values less than 0.05 were deemed to indicate statistical significance.

\section{RESULTS}

The participant characteristics are shown in Table 1. The children ranged in age from 1 to 11 years (mean age, 5.6 years); whereas 13 and 22 children had unilateral and bilateral spastic $\mathrm{CP}$, respectively. All 35 patients (19 boys) were spastic: 16 were classified as level I-III and 19 were classified as level IV-V on the GMFCS (Table 1).

To begin with, the R1 and R2 were significantly decreased after the 10-day break in PT (Table 2). It is noted that the range of dorsiflexion of the ankle joint decreased after the break significantly in both the knee flexion position (differences in R1 after the holidays, $-3.097^{\circ}$; $\mathrm{F}=16.87, \mathrm{p}<0.001$ and differences in $\mathrm{R} 2$ after the holidays, $\left.-5.353^{\circ} ; \mathrm{F}=12.84, \mathrm{p}=0.0004\right)$ and the knee extension position (differences in R1 after the holidays, $-3.504^{\circ}$; $\mathrm{F}=7.68, \mathrm{p}=0.0057$ and differences in $\mathrm{R} 1$ after the holidays, $-3.3316^{\circ} ; \mathrm{F}=18.9465, \mathrm{p}<0.0001$ ) (Fig. 1). The range of dorsiflexion of the ankle joint was not significantly different among the GMFCS levels in the knee flexion or extension positions (differences in $\mathrm{R} 1$ after the holidays, flexion $\mathrm{p}=0.3271$ and extension $\mathrm{p}=0.6863$; differences in $\mathrm{R} 2$ after

Table 2. Changes in ankle dorsiflexion angle

\begin{tabular}{lrrr}
\hline \multirow{2}{*}{ Knee position } & \multirow{2}{*}{ MTS } & \multicolumn{2}{c}{ Holiday } \\
\cline { 3 - 4 } & & \multicolumn{1}{c}{ Before } & \multicolumn{1}{c}{ After } \\
\hline Rt. flexion & R1 & $7.554 \pm 13.451$ & $1.667 \pm 16.257$ \\
& R2 & $21.643 \pm 13.110$ & $18.611 \pm 14.173$ \\
Rt. extension & R1 & $-3.250 \pm 15.215$ & $-7.806 \pm 17.094$ \\
& R2 & $11.393 \pm 15.229$ & $7.083 \pm 18.023$ \\
Lt. flexion & R1 & $11.115 \pm 14.668$ & $5.286 \pm 15.374$ \\
& R2 & $22.131 \pm 12.631$ & $18.143 \pm 15.109$ \\
\hline Lt. extension & R1 & $0.836 \pm 17.098$ & $-3.929 \pm 18.364$ \\
& R2 & $10.902 \pm 17.066$ & $6.786 \pm 19.375$ \\
\hline
\end{tabular}

Values are presented as mean \pm standard deviation and defined the neural ankle position as 0 and the dorsiflexion and the plantar flexion ankles were positive $(+)$ and negative (-), respectively.

MTS, Modified Tardieu Scale. 


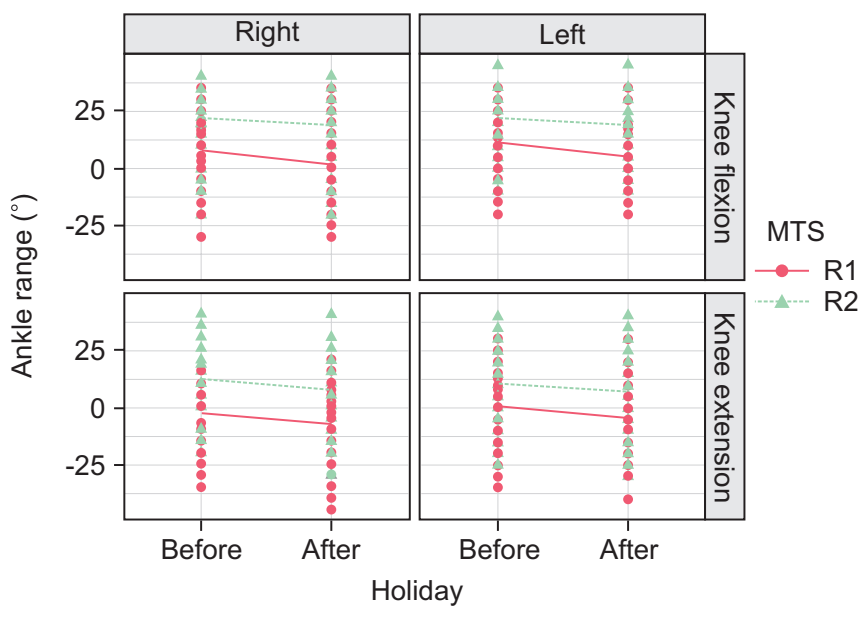

Fig. 1. The range of motion of ankle dorsiflexion decreased after the holiday compared to before the holiday period. The vertical axis of the graph indicates the ankle dorsiflexion angle ('+' dorsiflexion, '-' plantarflexion, ' 0 ' neutral position). The solid and dotted lines indicate mean values. MTS, Modified Tardieu Scale.

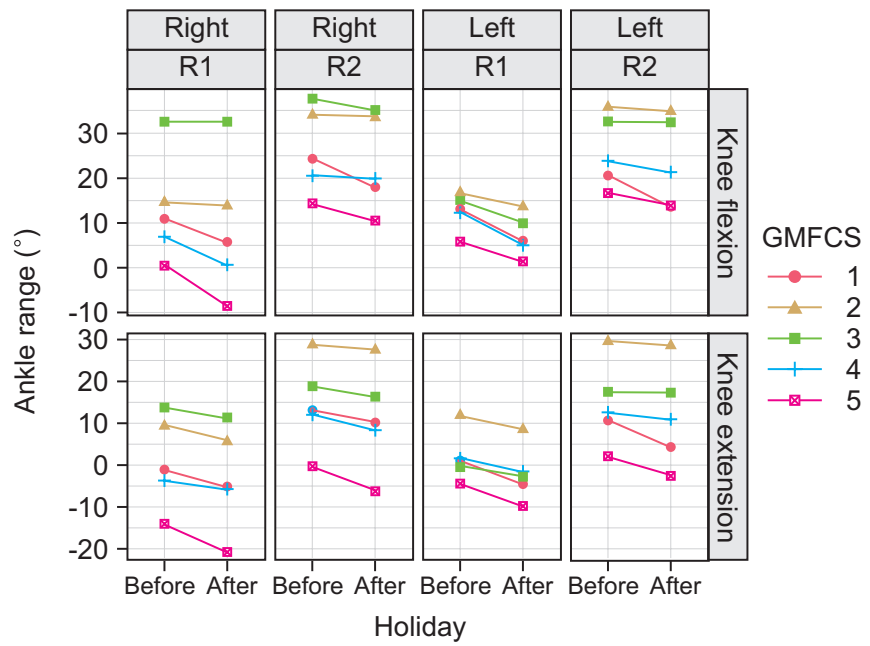

Fig. 2. The range of dorsiflexion of the ankle joint showed the decreasing tendency across the holiday regardless of GMFCS levels. The vertical axis of the graph indicates the ankle dorsiflexion angle ('+' dorsiflexion, '-' plantarflexion, ' 0 ' neutral position). The solid and dotted lines indicate mean value. GMFCS, Gross Motor Function Classification System.

the holidays, flexion $\mathrm{p}=0.3808$ and extension $\mathrm{p}=0.3536$ ) (Fig. 2). Moreover, R1 and R2 were not significantly different between the two GMFCS groups (Fig. 3). Similarly, the R1 and R2 values did not differ significantly according to sex (differences in R1 after the holidays, flexion $\mathrm{p}=0.9136$ and extension $\mathrm{p}=0.3468$; differences in $\mathrm{R} 2$ after

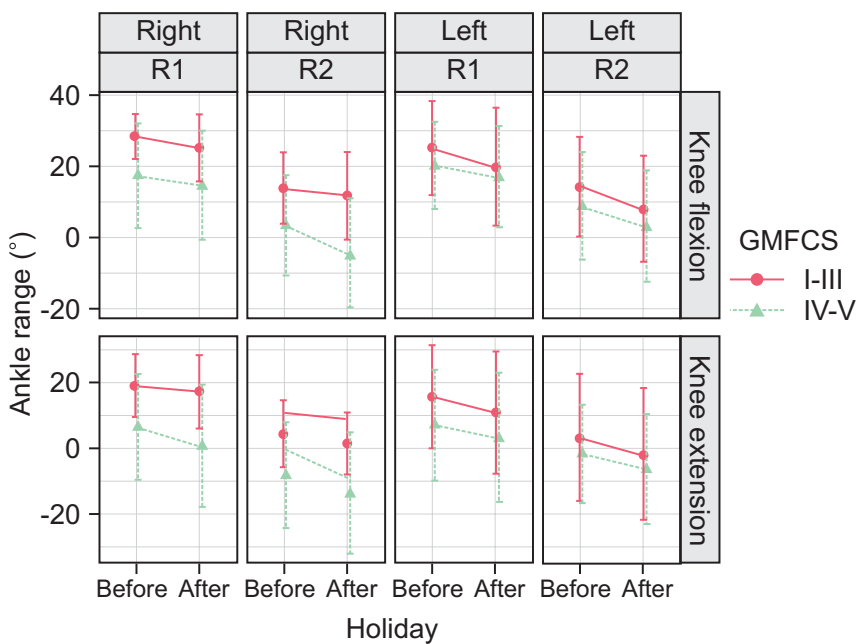

Fig. 3. Divided into two groups among GMFCS levels, IIII and VI-V, the range of dorsiflexion of the ankle joint was also decreased in the knee flexion and the knee extension, but no significant difference in decrement of the area of R1 and R2 is noted between the two groups. GMFCS, Gross Motor Function Classification System.

the holidays, flexion $\mathrm{p}=0.3156$ and extension $\mathrm{p}=0.2431$ ), age (differences in $\mathrm{R} 1$ after the holidays, flexion $\mathrm{p}=0.6841$ and extension $\mathrm{p}=0.3345$; differences in $\mathrm{R} 2$ after the holidays, flexion $\mathrm{p}=0.9582$ and extension $\mathrm{p}=0.9296$ ), or age group ( 4 years vs. 5 years, $\mathrm{p}>0.05$ ).

The difference between $\mathrm{R} 2$ and $\mathrm{R} 1$ (R2-R1) represents the dynamic component of spasticity. The R2-R1 measurement in the knee flexion position was significantly affected by interruption of PT ( $\mathrm{t}=2.301, \mathrm{p}=0.042)$; however, R2-R1 in the knee extension position did not change significantly in the absence of PT $(t=-0.260, p=0.7615)$.

\section{DISCUSSION}

Our findings suggest that regular PT may be involved in maintaining the range of dorsiflexion of the ankle joint, and in the control of ankle plantarflexor muscle tone in children with spastic CP. Our findings suggest that dynamic ankle plantarflexor tone in the knee flexion position may increase following the interruption of regular PT in the pediatric patient population. Because physical, occupational, and speech and language therapies are viewed as essential for the management of patients with CP [12], it is not practical or ethical to design a study with a no-therapy control condition. Furthermore, comparisons between children who receive regular therapy and 
those who do not are replete with confounding factors. It is emphasized that our study is the first to investigate the effects of disrupted regular or intensive PT over a 10-day period.

Several previous studies have investigated the treatment of hypertonia; however, a positive effect of PT has not been clearly demonstrated. Pin et al. [17] argued that passive stretching alone was not sufficient to preserve muscle length, and recommended the addition of orthoses or casts to increase the effectiveness of stretching programs. However, some studies have confirmed the clinical assumption that PT can alleviate spasticity and prevent contracture in children with CP [18-21]. Damiano [20] found that the use of progressive strength training exercises needed to be performed on a regular basis to retain the benefits of improved muscle strength and to improve walking ability without increased spasticity. Our findings on the effects of an interruption in PT interventions for spasticity may facilitate the development of appropriate rehabilitation strategies for children with CP.

There are no specific guidelines on the duration and frequency of PT have been established for children with CP possibly because of the heterogeneity and wide variation in severity among this pediatric population demographic. In what follows, it is seen that intensive therapy more than three times a week has been shown to have a significant impact on gross motor function in children affected with this condition [22,23]. A previous study found that strength training two to three times a week on nonconsecutive days for a minimum of 8 weeks was sufficient to increase strength for these children [24]. There are no standard recommendations for the duration and frequency of stretching exercises to be performed $[17,18,25]$. In this respect, a 30-minute exercise regimen is common to use and stretching one to three times a week has been shown to decrease spasticity in children with CP [18]. The patients in our study had received PT from one to six times a week in several different institutions. Because of the lack of information about current therapies for all patients, we were not able to assess the influence of frequency or duration of therapy. We found that the angle of the ankle joint dorsiflexion during fast-velocity (R1) and slow-velocity (R2) stretches decreased when PT was not performed over a 10-day interval. This finding suggests that regular PT sessions are necessary to maintain ROM in spastic ankle joints, which may ultimately be helpful in prevention of the deterioration of motor function in children with spasticity.

Although the mechanisms underlying spasticity are not well understood, neuroplasticity in the central motor pathways and the mechanical components of the muscle are critical factors in changes in muscle tone and stiffness in patients with spastic CP. In terms of neuroplasticity, exercise interventions may change muscle tone in response to tonic reflex activity allowing an increased ROM for the fast stretch [26]. Furthermore, stretching can alter the mechanical properties of the muscle, which may increase muscle or fascicle length [27]. In this context, it is seen that shortened muscles potentiate the stretch reflex setting in motion a vicious cycle in which increasing spasticity leads to muscle contracture, which in turn potentiates the stretch reflex [28].

Spasticity and contracture of the gastrocnemius muscles interfere with ambulation eventually resulting in toe walking and other gait problems. Spasticity tends to decrease in children between the ages of 4 and 12 years, which may explain the clinical observation that some patients with CP begin to walk with heel contact if the gastrocnemius muscle is not deformed [28]. Taking this into account, young children with spastic CP must be carefully managed to prevent muscle shortening, until they are at the age when spasticity may decrease naturally. Thus, our findings are important for patients with CP under 12 years of age, in whom prevention of ankle joint deterioration is critical. Furthermore, we found that there were no significant age-related effects of interrupted exercise on ankle ROM and spasticity in children under 12 years of age.

Previous studies have shown that the medial gastrocnemius muscle volume and angle of ankle dorsiflexion were decreased in children with spastic CP [27,29]; however, the plantar flexor strength training would result in increases in medial gastrocnemius muscle volume and angle of the ankle dorsiflexion [27]. Thus, the additional benefits of PT including restoring the balance between muscle volume and strength as well as spasticity control should not be overlooked as beneficial in these participants or similarly situated individuals. Interventions such as botulinum toxin injection, oral medication, and orthopedic surgery may lead to inadequate volumetric muscle growth in individuals with neurodisabilities. Although the evidence suggests that these interventions 
reduce spasticity reducing muscle tone does not always improved motor function and walking ability.

Our study had several limitations. First, the outcome measures were not assessed using the Modified Ashworth Scale (MAS), which is commonly used to assess spasticity in individuals. Because our study was retrospective, we chose to assess the parameter with the most data have. However, measurement with MAS is influenced by ROM limitations and it is difficult to interpret differences in MAS scores in shortened muscles, which may limit interobserver reliability [30]. Gracies et al. [16] reported that the MTS had excellent intra-rater and inter-rater reliability when assessing the elbow and ankle joints of children with CP. Although all of the participants in our study were children with spastic $\mathrm{CP}$, the participants had varying degrees of muscle tone among different muscles, and it was noted that their motor abilities also varied widely among individual participants. Given this heterogeneity, a subgroup analysis may have provided useful information; however, because we did not have a sufficient number of participants for subgroup analysis, we assessed the effects of age and GMFCS levels on the range of ankle motion. Thus, the retrospective design and relatively small sample size are further limitations of our study. The finding that the difference between R2 and R1 (R2R1) after the 10-day PT break showed a slight decrease in the knee flexion position, whereas the difference in the knee extension position did not reach statistical significance, raises the possibility of type II error ( $\beta$ error) due to the small sample size. However, there is no precedent for studies such as ours due to the ethical problem of discontinuing basic management in children with $\mathrm{CP}$. The lack of comparative study with the control group is also an important limitation of this study. Since there was no comparative evaluation of the pre and post MTS during the period of receiving the regular PT in the study, the time effect could not be clearly excluded. A further study is needed to compare this result and conclusion with the control group. Although a weekday existed during a lengthy public holiday, there was the tendency of many institutions and families to have their holiday on that weekday. However, it was difficult to identify whether PT was performed and participated in at other institutions on that day, which could be a limitation of the study. Finally, the long-term follow-up evaluation of patients was not done. Further prospective longitudinal studies with larger groups are needed to determine the long-term trajectory of spasticity and ROM with ongoing therapy.

It is well known that muscle tone and ROM of the ankle joints are important for the maintenance of standing posture and ambulatory function. The effects of PT on spasticity are still under discussion, and no definite regimen has been established for PT. Our findings suggest that the cessation of PT for about 10 days during a holiday period may significantly affect the flexibility or muscle tone and ROM of the spastic ankle joint. In addition, during the holiday period, family home care may have been stopped due to the family events and trips. For this reason, it is noted that regular PT to prevent ankle contracture and control hypertonicity is an important component of the care continuum for children with spastic CP.

\section{CONFLICT OF INTEREST}

No potential conflict of interest relevant to this article was reported.

\section{ACKNOWLEDGMENTS}

This research was supported by Basic Science Research Program through the National Research Foundation of Korea (NRF) funded by the Ministry of Science, ICT \& Future Planning (No. NRF-2017R1C1B5017423).

\section{AUTHOR CONTRIBUTION}

Conceptualization: Hong BY, Kim JS. Methodology: Hong BY, Kim EK, Son DB. Formal analysis: Hwang Y, Hong BY. Funding acquisition: Hong BY. Project administration: Hong BY, Kim EK, Son DB. Visualization: Hwang Y, Sul B, Hong BY. Writing - original draft: Lee H, Hong BY. Writing - review and editing: Lee H, Hong BY, Lim SH. Approval of final manuscript: all authors.

\section{REFERENCES}

1. Bax M, Goldstein M, Rosenbaum P, Leviton A, Paneth N, Dan B, et al. Proposed definition and classification of cerebral palsy, April 2005. Dev Med Child Neurol 2005;47:571-6.

2. Surveillance of Cerebral Palsy in Europe. Surveillance of cerebral palsy in Europe: a collaboration of cerebral 
palsy surveys and registers. Dev Med Child Neurol 2000;42:816-24.

3. Westbom L, Rimstedt A, Nordmark E. Assessments of pain in children and adolescents with cerebral palsy: a retrospective population-based registry study. Dev Med Child Neurol 2017;59:858-63.

4. Yim SY, Yang CY, Park JH, Kim MY, Shin YB, Kang EY, et al. Korean Database of Cerebral Palsy: a report on characteristics of cerebral palsy in South Korea. Ann Rehabil Med. 2017;41:638-49.

5. Scholtes VA, Becher JG, Beelen A, Lankhorst GJ. Clinical assessment of spasticity in children with cerebral palsy: a critical review of available instruments. Dev Med Child Neurol 2006;48:64-73.

6. Sees JP, Miller F. Overview of foot deformity management in children with cerebral palsy. J Child Orthop 2013;7:373-7.

7. Hagglund G, Lauge-Pedersen H, Wagner P. Characteristics of children with hip displacement in cerebral palsy. BMC Musculoskelet Disord 2007;8:101.

8. Hagglund G, Wagner P. Development of spasticity with age in a total population of children with cerebral palsy. BMC Musculoskelet Disord 2008;9:150.

9. Chan G, Miller F. Assessment and treatment of children with cerebral palsy. Orthop Clin North Am 2014; 45:313-25.

10. Gormley ME Jr. Treatment of neuromuscular and musculoskeletal problems in cerebral palsy. Pediatr Rehabil 2001;4:5-16.

11. Nahm NJ, Graham HK, Gormley ME Jr, Georgiadis AG. Management of hypertonia in cerebral palsy. Curr Opin Pediatr 2018;30:57-64.

12. Colver A, Fairhurst C, Pharoah PO. Cerebral palsy. Lancet 2014;383:1240-9.

13. Wiart L, Darrah J, Kembhavi G. Stretching with children with cerebral palsy: what do we know and where are we going? Pediatr Phys Ther 2008;20:173-8.

14. Wood E, Rosenbaum P. The gross motor function classification system for cerebral palsy: a study of reliability and stability over time. Dev Med Child Neurol 2000;42:292-6.

15. Katusic A, Alimovic S. The relationship between spasticity and gross motor capability in nonambulatory children with spastic cerebral palsy. Int J Rehabil Res 2013;36:205-10.

16. Gracies JM, Burke K, Clegg NJ, Browne R, Rushing C,
Fehlings D, et al. Reliability of the Tardieu scale for assessing spasticity in children with cerebral palsy. Arch Phys Med Rehabil 2010;91:421-8.

17. Pin T, Dyke P, Chan M. The effectiveness of passive stretching in children with cerebral palsy. Dev Med Child Neurol 2006;48:855-62.

18. Park EY, Kim WH. Effect of neurodevelopmental treatment-based physical therapy on the change of muscle strength, spasticity, and gross motor function in children with spastic cerebral palsy. J Phys Ther Sci 2017;29:966-9.

19. Tilton AH. Therapeutic interventions for tone abnormalities in cerebral palsy. NeuroRx 2006;3:217-24.

20. Damiano DL. Rehabilitative therapies in cerebral palsy: the good, the not as good, and the possible. J Child Neurol 2009;24:1200-4.

21. Wu YN, Hwang M, Ren Y, Gaebler-Spira D, Zhang LQ. Combined passive stretching and active movement rehabilitation of lower-limb impairments in children with cerebral palsy using a portable robot. Neurorehabil Neural Repair 2011;25:378-85.

22. Arpino C, Vescio MF, De Luca A, Curatolo P. Efficacy of intensive versus nonintensive physiotherapy in children with cerebral palsy: a meta-analysis. Int J Rehabil Res 2010;33:165-71.

23. Hong BY, Jo L, Kim JS, Lim SH, Bae JM. Factors influencing the gross motor outcome of intensive therapy in children with cerebral palsy and developmental delay. J Korean Med Sci 2017;32:873-9.

24. Verschuren O, Peterson MD, Balemans AC, Hurvitz EA. Exercise and physical activity recommendations for people with cerebral palsy. Dev Med Child Neurol 2016;58:798-808.

25. O'Dwyer N, Neilson P, Nash J. Reduction of spasticity in cerebral palsy using feedback of the tonic stretch reflex: a controlled study. Dev Med Child Neurol 1994; 36:770-86.

26. Aisen ML, Kerkovich D, Mast J, Mulroy S, Wren TA, Kay RM, et al. Cerebral palsy: clinical care and neurological rehabilitation. Lancet Neurol 2011;10:844-52.

27. McNee AE, Gough M, Morrissey MC, Shortland AP. Increases in muscle volume after plantarflexor strength training in children with spastic cerebral palsy. Dev Med Child Neurol 2009;51:429-35.

28. Hagglund G, Wagner P. Spasticity of the gastrosoleus muscle is related to the development of reduced pas- 
sive dorsiflexion of the ankle in children with cerebral palsy: a registry analysis of 2,796 examinations in 355 children. Acta Orthop 2011;82:744-8.

29. Bell KJ, Ounpuu S, DeLuca PA, Romness MJ. Natural progression of gait in children with cerebral palsy. J Pediatr Orthop 2002;22:677-82.
30. Numanoglu A, Gunel MK. Intraobserver reliability of modified Ashworth scale and modified Tardieu scale in the assessment of spasticity in children with cerebral palsy. Acta Orthop Traumatol Turc 2012;46:196200. 